\title{
Photonics for net zero
}

\author{
John R. Lincoln \\ Photonics Leadership Group and Harlin Ltd., Salisbury, United Kingdom
}

Achieving net zero will be the biggest technological challenge of the $21^{\text {st }}$ century. A truly global, all-pervasive challenge impacting every application, market, and technology. There are no guidelines, instructions, or silver bullets that provide overnight solutions. Achieving net zero will be a sum of countless innovations across energy generation, resource efficiency, and waste reduction. Photonics will have a pivotal role in all of these; directly, by reducing the carbon impact from using light-based technologies; and indirectly, through increased use of photonics in monitoring and reduction in greenhouse gasses across a vast range of industries.

Addressing any challenge starts with recognizing the problem, before setting targets for resolution to stimulate and inspire solutions. The 2015 Paris Climate Accords marked a discernible movement along this process. The COP26 summit illustrates how the agenda has now shifted from recognition to targets, with particular targets set for achieving net zero $\mathrm{CO}_{2}$ emissions. Yet these targets are set for 2035, 2040, 2050, and beyond-all timescales in the distant future, compared to the cycles of technology innovation, politics, or corporate strategy. Why should participants in a fast-moving technology sector like photonics care today?

Just as investment markets move on information, or rumours of information, without waiting for physical events, movement on sustainability is starting in companies now that those long-distant targets have been set. Look at almost any annual report from a listed company and the first dozen pages are full of references to sustainability and assessments of current climate/ $\mathrm{CO}_{2}$ impact-large companies are not waiting for 2035. A random sample of annual reports of firms associated with the photonics industry shows a 7-fold increase in terms associated with sustainability (climate, energy etc) between 2016 and 2020. Sustainability, often in combination with responsibility, is already getting significant corporate attention, at least in reporting.

Why? Partly, companies are responding to the interests of investors who are under pressure to hold more sustainable portfolios. But, more importantly, they are starting to respond to customer demand. Once a major listed company at the top of the supply chain has assessed their own carbon footprint, it is small step to ask the same of their suppliers. To keep customers, suppliers are having to assess the direct impact of their products and operations, with such requests rapidly cascading down through the supply chain and predictably arriving at photonics companies. The most forward-looking naturally act before they are asked, anticipating demand. Starting first in easy areas, such as energy efficiency and recycled/reused packaging content, such requests will inevitably lead more to more complex quantification of lifetime energy use and full lifecycle of embedded carbon.

The next question is inevitable- -can you improve?" For many, sustainability will rapidly become a $3^{\text {rd }}$ axis of differentiation alongside cost and performance, a method of differentiating, winning contracts, and outperforming the competition. Not everyone will choose to compete on this new dimension, but it will generate new competitive threats and opportunities in photonics so that we will all need to give sustainability its full attention. Combined with increasing focus on

(C) The Authors. Published by SPIE and CLP under a Creative Commons Attribution 4.0 International License. Distribution or reproduction of this work in whole or in part requires full attribution of the original publication, including its DOI.. [DOI: 10.1117/1 .AP.4.1.010501]

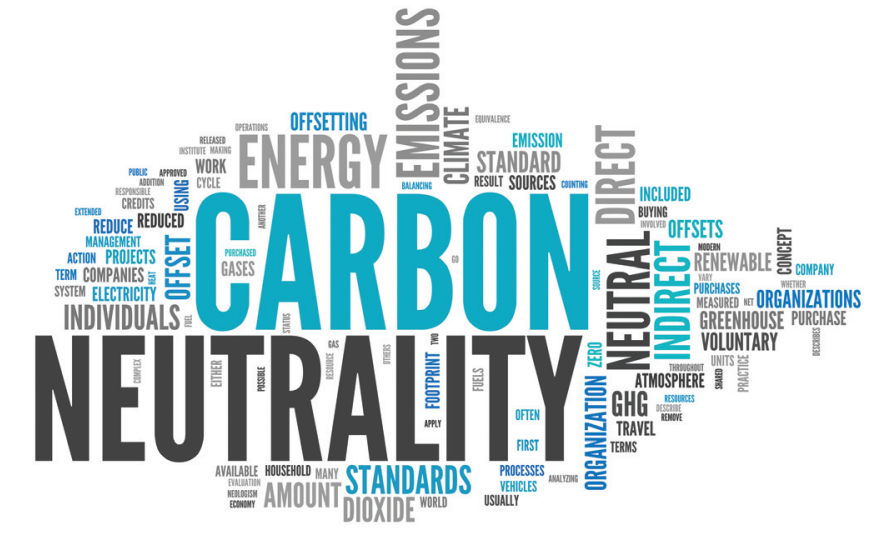

Achieving carbon neutrality, a.k.a. "net zero," will be the biggest technological challenge of the $21^{\text {st }}$ century. Image credit: Shutterstock (179238317).

resilience and point of origin, sustainability should also provide a welcome alternative to negative spirals of price-based competition. Such competitive cycles will drive corporate behavior and innovation far in advance of those 2050 targets as they impact the ability to win customers and make profits, not just in the distant future, but in the near term, too.

If increasing sustainability impacts near-term behavior, how and where does photonics take advantage of the opportunities such shifts will provide? As a crosscutting enabling technology the countless areas photonics can contribute can be grouped into three distinct areas: generation, consumption, and waste.

\section{Generation}

Direct energy generation through photovoltaics (PV) is the most obvious contribution photonics can make to achieving net zero. There are few more pure forms of photonics than the generation of electricity from sunlight absorbed through a semiconductor junction. Advances in photonics will incrementally increase the efficiency of silicon PV, and potentially make compound semiconductor PV viable. Novel materials, such as perovskites, will also lead to flexible PV enabling greater building integration, increasing the surface area generating electricity globally without compromising architectural design.

Photonics can also make a significant contribution to other methods of power generation, helping to maximize the output of both traditional sources (e.g., gas turbines) and renewables (e.g., wind generation). Using photonics to increase the energy efficiency of the installed base of wind turbines by just $1 \%$ would generate enough additional power by 2035 to save building five nuclear power stations. ${ }^{1}$ That $1 \%$ can be from LIDAR mapping of wind to optimize turbine location and blade pitch in operation, and/or from the more mundane optical monitoring of gear box oil to reduce service times. The photonics contribution does not have to be in the headlines; it can, as is so often the case with an enabling technology, be embedded and hidden, making an invaluable contribution to a much bigger system. 


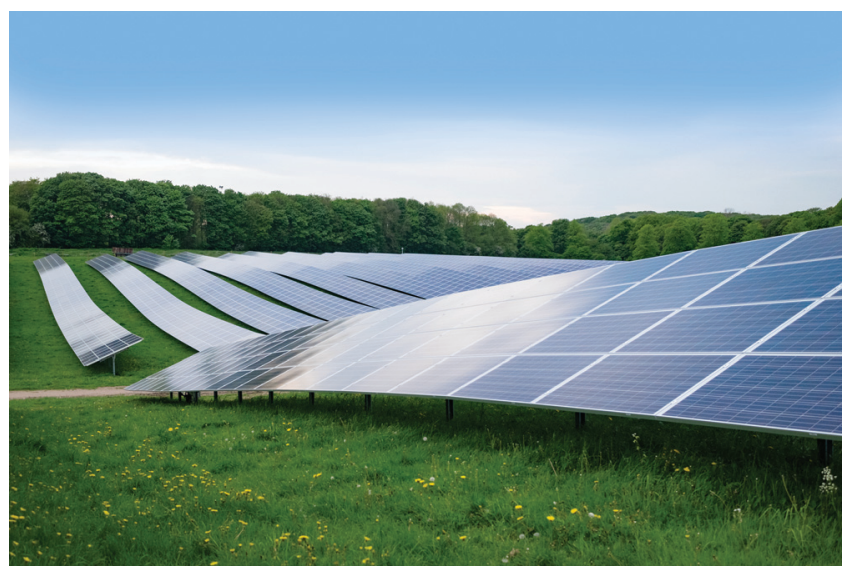

There are few more pure forms of photonics than the generation of electricity from sunlight absorbed through a semiconductor junction. Advances in photonics will incrementally increase the efficiency of silicon PV, and potentially make compound semiconductor PV viable. Image credit: Shutterstock (1115812136).

The photonics impact does not stop at the generation of grid connected power. As emphasized in the International Year of Light, off-grid PV is vital for improving education in developing countries, enabling study after dark without polluting kerosene lamps, with an immediate impact on improving health as well as longer term impact on the planet.

PV panels are also not the only mechanism through which photonics can harness the power of the sun. Photocatalysis and photo-electrocatalysis have the potential to make significant improvements to the efficiency of chemical processing, directly reducing the energy inputs required as we seek cleaner fuels and a more sustainable chemical and pharmaceutical industries.

\section{Consumption}

With light emission from LEDs and semiconductor lasers leveraging the same physics as $\mathrm{PV}$, volume applications involving the generation

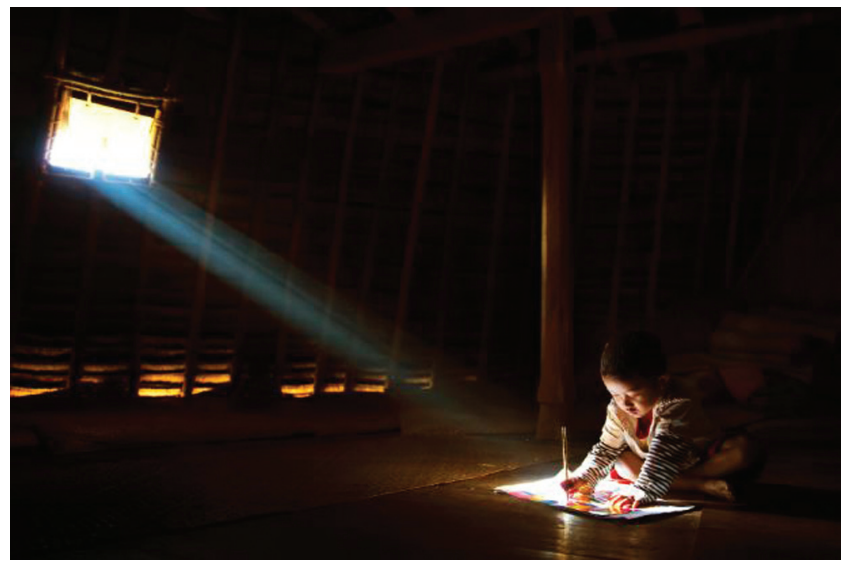

As emphasized in the International Year of Light, off-grid PV is vital for improving education in developing countries, enabling study after dark without polluting kerosene lamps, with an immediate impact on improving health as well as longer term impact. Image credit: Handi Laksono, winner of the 2015 SPIE International Year of Light Photo Contest People's Choice Award. of light are naturally energy consumers, sometimes significantly so. Optical communications dominates the transport of data from the cell phone/mobile mast, WiFi box, or street cabinet, not just to datacenters, but increasingly within datacenters, to the processors, storage, and memory arrays that are the engines of the digital economy. Networks use close to $1 \%$ of global electricity; datacenters, $4 \%$. Energy consumption per bit of data has therefore become a major focus of the integrated photonics that forms the heart of such systems.

The challenge in optical networking is not current consumption levels, but the direction of travel. Digital applications are booming-from industry 4.0 to autonomous vehicles and entertainment-our appetite for data seems neverending. Just as we are trying to reduce our energy consumption, the digital economy is consuming ever more power.

The nature of our interaction with data is also changing, from one-directional consumption (e.g., streaming) to bidirectional interactivity in gaming, remote surgery, finance, video calls, etc. The digital economy will increasingly depend as much on latency as it does bandwidth. Those latency demands also mean past solutions, such as placing datacenters far from users where sustainable power and cooling is abundant, e.g., adjacent to hydroelectric plants in the colder latitudes, will no longer work. As photonics innovators, we now need work harder than ever, so the efficiency of optical communications improves faster than combined bandwidth and latency capability. No mean feat when internet traffic is growing at $26 \%$ a year. ${ }^{2}$

The photonics role in consumption does not end with data. Lighting recently accounted for $15 \%$ of the electricity consumed by residential and commercial users in the USA. ${ }^{3}$ The transformation to highly efficient LED lighting is already well underway helping to reduce this. While further efficiency gains may well be possible, perhaps greater future sustainability impacts will be in smart lighting. Adjusting artificial light levels in intensity and color, to complement and top-up that available naturally, will support sustainability and also improve our overall well-being.

When most people worked in an office or factory, the pathway to realization, at least in a corporate setting, was perhaps clear, if not simple to quantify, combining gains in employee welfare, productivity, and energy efficiency. In a world where many of us will continue to work from home for the long term, such smart lighting must be delivered to a much more complex and distributed set of users. The very variation in home working setups and the need to change from office-by-day to home-by-night, makes the logic of smart lighting yet more compelling.

\section{Waste}

At the simplest level much of photonics involves optical surfaces and fragile components that are sensitive to degradation and damage. We are used to packing optics in multiple protective layers, often with unsustainable plastic. As an industry, we can both make those optical surfaces and components more durable and use more sustainable packaging solutions. We must avoid excessive caution and realize when less is more, e.g., why does a mobile phone with a scratch-resistant glass screen still come with a protective plastic film?

But the photonics impact on reducing waste will be much greater. Photonics will be vital to the circular economy. Recycling, and even more so repurposing, depends on being certain about the material or product you are starting with at a level far beyond how a product is visibly labelled. If sustainable materials are valuable, fakes will proliferate - up to 1 in 20 of the original pound coins in circulation in the UK were estimated to be fakes. ${ }^{4}$

Multiple forms of spectroscopy can provide detailed matching of materials against libraries of expected (and unexpected compositions) quickly and nondestructively. This will be vital for the verification of 


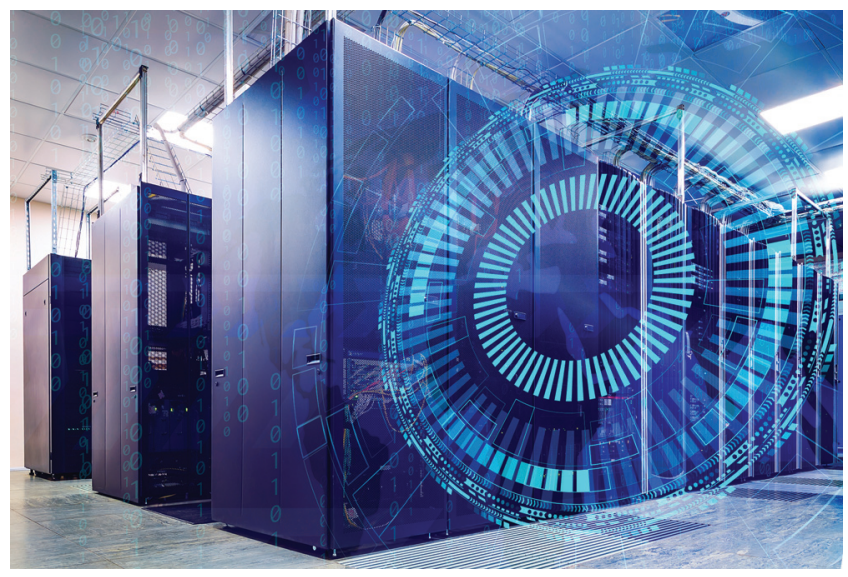

As photonics innovators, we now need work harder than ever, so the efficiency of optical communications improves faster than combined bandwidth and latency capability. Image credit: Shutterstock (543151186).

more sustainable materials-what better than photonics to distinguish two visually identical steels, one made in a high-efficiency, highly sustainable process, the other from a less efficient more polluting source? Such forms of verification and traceability will become essential to maintaining the integrity and smooth operation of sustainable supply chains.

The best way to avoid end-of-life impact is of course to increase product lifetimes and to delay the time at which a product no longer delivers customer benefit. Within photonics, innovative adaptable, reconfigurable products, e.g., combining metastable/phase change materials in integrated photonics, will have a key role. Adaptability will enable one product to have many applications, maximizing yield and reducing waste at the start of life, and enabling through-life reconfiguration to prolong useful product lifetimes.

Consideration of lifetime also leads to a potential paradox between lifetime and efficiency: if a photonics innovation is more energy efficient, but has a shorter lifetime, does it make a positive or negative impact on net zero? Assessing full life-cycle carbon impact versus operational impact would be a first step, and something photonics innovators will increasingly need to consider. However, the challenge is more complex when lifetime and efficiency are dependent on how photonics is integrated and when different organizations are responsible for the manufacture and operation.

In summary, photonics will have a major impact in delivering net zero addressing this biggest of challenges on many fronts. Indeed, as momentum builds, it is vital that photonics is part of the solution.

Aside from contributing to saving the planet, as an industry which continues to grow at over $8 \%$ a year ${ }^{5}$ we need to continue to attract the very best talent. Many of the next generation of employees are already making decisions on which college or university they attend according to the sustainability credentials of different institutes. Soon this environmentally conscious generation will be starting their careers and smart enough to spot simple green washing. If photonics wishes to attract the very best talent in increasing numbers to our $\mathrm{PhD}$ programs and our companies, we must be visibly active in generating sustainable solutions. If not, we will be part of the problem, unable to attract the best, hampering growth, hampering impact, and making it harder to achieve net zero.

\section{References}

1. H. Bookey, Private Communication, Fraunhofer Centre for Applied Photonics (2021).

2. Cisco, "Global 2021 forecast highlights," VNI Complete Forecast Highlights, https://www.cisco.com/c/dam/m/en_us/solutions/ service-provider/vni-forecast-highlights/pdf/Global_2021_Forecast_ Highlights.pdf (accessed December 2021).

3. National Academies of Science, Engineering, and Medicine, "What you need to know about energy," http://needtoknow.nas .edu/energy/energy-efficiency/lighting/ (accessed December 2021).

4. B. Ando, "Fake $£ 1$ coin estimate 'doubled'," BBC News, 8 April 2009, http://news.bbc.co.uk/1/hi/7988001.stm (accessed December 2021).

5. SPIE, "2020 Optics and Photonics Industry Report (Fall Update)," 25 January 2021, https://spie.org/news/2020-optics-and-photonicsindustry-report (accessed December 2021). 